\title{
Rare presentation of intractable tuberculous panophthalmitis with intraocular and intraorbital abscesses: a case report
}

\author{
Sutasinee Boonsopon ${ }^{1}$, Nattaporn Tesavibul ${ }^{1}$, Mongkol Uiprasertkul², Supinda Leeamornsiri ${ }^{3}$ \\ and Pitipol Choopong ${ }^{1 *}$ (iD
}

\begin{abstract}
Background: We report a rare presentation of extrapulmonary tuberculosis.

Case presentation: A 29-year-old Burmese woman with human immunodeficiency virus infection and known pulmonary tuberculosis who had been treated for 5 months presented to our hospital with unilateral progressive painful visual loss of 1 month's duration. She was diagnosed with tuberculous panophthalmitis with subretinal and intraorbital abscesses, conjunctival abscess, and extraocular muscle tuberculoma. The diagnosis was confirmed by a conjunctival pus swab with a positive result for acid-fast bacilli and a positive result for a mycobacterial culture. There was high suspicion of multidrug-resistant tuberculosis. Despite receiving ongoing aggressive treatment with conventional antituberculous medications, this patient required subtotal orbital exenteration to control her infection and prevent further progression. Second-line antituberculous medications were added to the first-line therapy, with satisfactory results achieved.

Conclusions: Tuberculous panophthalmitis with intraocular and intraorbital abscesses is a rare presentation of extrapulmonary tuberculosis. Patients who do not respond to first-line antituberculous therapy might be infected with either single-drug or multidrug-resistant Mycobacterium tuberculosis. Patient compliance is one of the key factors that can alter the course of treatment. Careful patient monitoring can improve disease progression, outcome, and prognosis.
\end{abstract}

Keywords: Tuberculous panophthalmitis, Tuberculous orbital abscess, Extrapulmonary tuberculosis

\section{Background}

Mycobacterium tuberculosis (Mtb) is an obligate pathogenic bacterial species in the family Mycobacteriaceae. Inhalation of this organism causes pulmonary infection. Hematogenous spread as a result of a ruptured pulmonary granuloma leads to multiple organ involvement, including the eye [1]. Failure to identify tuberculous mycobacterial ocular infection can lead to permanent visual damage, with uncontrolled infection potentially resulting in death [2]. We report a rare and serious case of a patient with

\footnotetext{
*Correspondence: pitipol.cho@mahidol.edu

'Department of Ophthalmology, Faculty of Medicine, Siriraj Hospital, Mahidol University, 2 Wanglang Road, Bangkoknoi, Bangkok 10700, Thailand Full list of author information is available at the end of the article
}

extrapulmonary tuberculosis (EPTB) who presented with tuberculous panophthalmitis with intraocular and intraorbital abscesses, despite ongoing aggressive treatment with conventional antituberculous therapy (ATT).

\section{Case presentation}

A 29-year-old Burmese woman with human immunodeficiency virus (HIV) infection who had been diagnosed with pulmonary tuberculosis was initially treated with isoniazid $300 \mathrm{mg} /$ day, rifampicin $450 \mathrm{mg} /$ day, pyrazinamide (unknown dosage), and ethambutol $800 \mathrm{mg} /$ day for 2 months. After a 2-month course of this four-drug regimen, pyrazinamide was discontinued. Five months after the initiation of ATT, the patient developed pain, 
redness, and blurred vision in her right eye. Two weeks later, her right eye became no light perception and exhibited proptosis with the presence of purulent discharge. Orbital computed tomography (CT) showed large intraconal, periorbital, and preseptal abscesses. She was then admitted to the primary care hospital and was treated for orbital cellulitis with a 1 -g ceftriaxone infusion twice daily. Oral ciprofloxacin $500 \mathrm{mg}$ twice daily was added 4 days later. Tobramycin eye drops $0.3 \%$ four times daily and tobramycin eye ointment twice daily were given.

The result of a conjunctival pus swab was positive for $1+$ acid-fast bacilli (AFB), and a culture revealed clusters of Staphylococcus spp. Despite an escalation of ATT and antibiotics for 1 week, the patient's clinical condition continued to worsen. She was then referred to our institution, Siriraj Hospital, Thailand's largest universitybased tertiary referral center. Upon arrival, her right eye showed marked chemosis with a large subconjunctival inferotemporal abscess (Fig. 1a). She had 3+ anterior chamber cells, mutton-fat keratic precipitates, ectropion uvea, and iris neovascularization. Exophthalmometry at 104-mm base showed a protrusion of the right eye of $20 \mathrm{~mm}$, compared with $13 \mathrm{~mm}$ in the left eye. The right lateral gaze and down gaze of her right eye were limited at 5 and 10 degrees, respectively. A dilated fundus examination showed a dense vitreous hemorrhage. An unclear subretinal lesion was identified. Her left eye was unremarkable. B-scan ultrasonography of her right eye showed an irregular globe contour and inhomogeneous vitreous echogenicity. A $13.3 \mathrm{~mm} \times 15 \mathrm{~mm}$ mass with a high initial A-scan spike and low to moderate internal reflectivity was identified underneath a high A-scan spike membrane-like lesion that extended beyond the globe into the orbital cavity (Fig. 1b).

Orbital CT was repeated, which showed an intraocular multiloculated ring contrast-enhanced mass that extended to the right orbit (Fig. 1c and d). The optic nerve, lateral rectus muscle, and inferior rectus muscle of the right eye were enlarged. The conjunctival pus swab was repeated, which stained positive for AFB. The patient's CD4 cell count was 123 cells $/ \mathrm{mm}^{3}$. An infectious disease (ID) specialist immediately ordered second-line ATT (750-mg amikacin infusion, 500-mg levofloxacin infusion, oral clarithromycin $1 \mathrm{~g} /$ day, and para-aminosalicylic acid $8 \mathrm{~g} /$ day). With the fact that the infection was intense and was not responding to conventional ATT, despite the previous 5 months of ATT treatment. The risk of progression to the central nervous system was weighed against preservation of the eyeball. With a discussion between the patient and the medical team, a decision was made to perform subtotal orbital exenteration. A large number of scleral and intramuscular tuberculomas were identified. One large subretinal abscess $(14 \mathrm{~mm} \times 10 \mathrm{~mm} \times 10 \mathrm{~mm})$ was present that extended to the orbital cavity through a scleral perforation. Pathological study of exenterated tissue

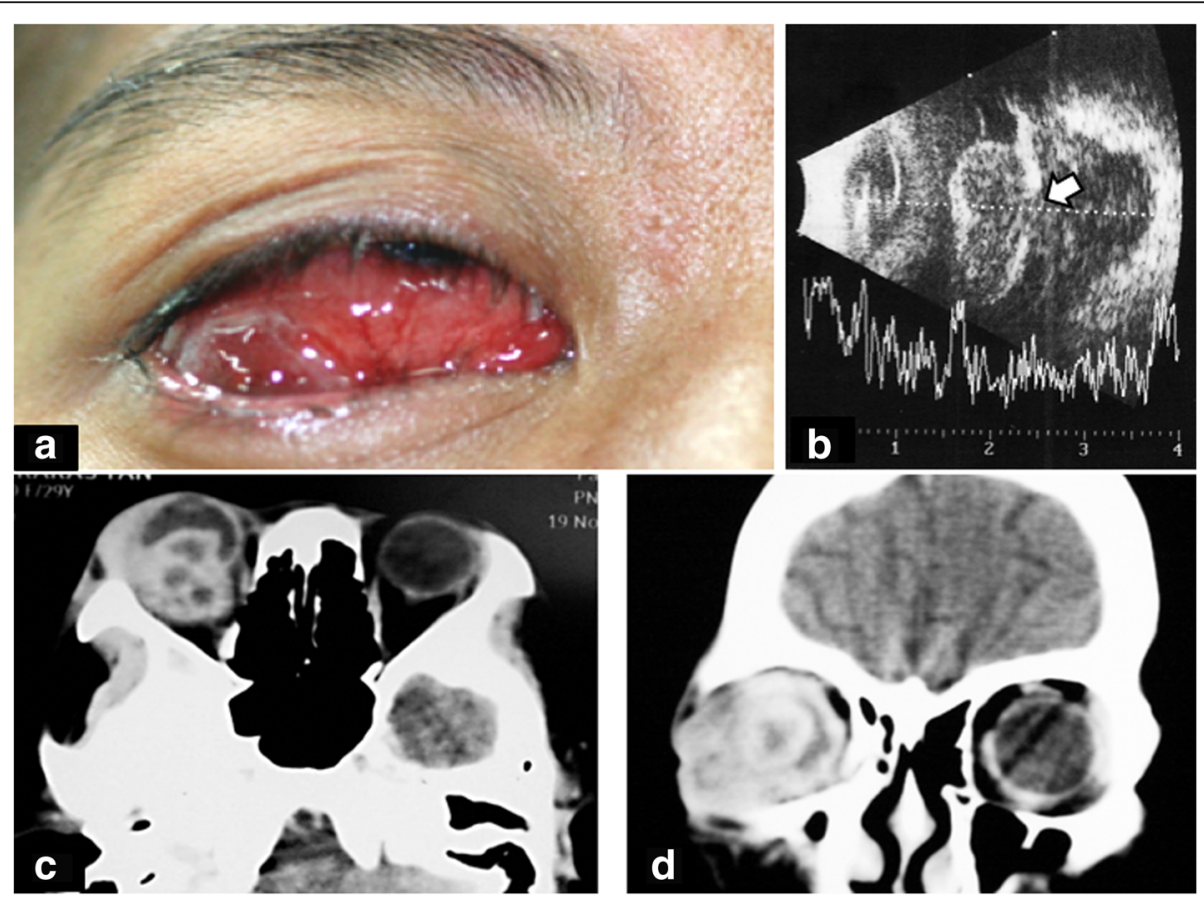

Fig. 1 a A large subconjunctival abscess is shown in the right eye inferotemporally with purulent discharge and chemosis. $\mathbf{b}$ B-scan ultrasonogram of the right eye shows a large intraocular mass extending beyond the globe through a scleral defect (arrow) into the orbital cavity. $\mathbf{c}$ and $\mathbf{d}$ Orbital computed tomographic scan shows in more detail the conjoint intraocular and intraorbital multiloculated, multiple-ring, contrast-enhanced mass 


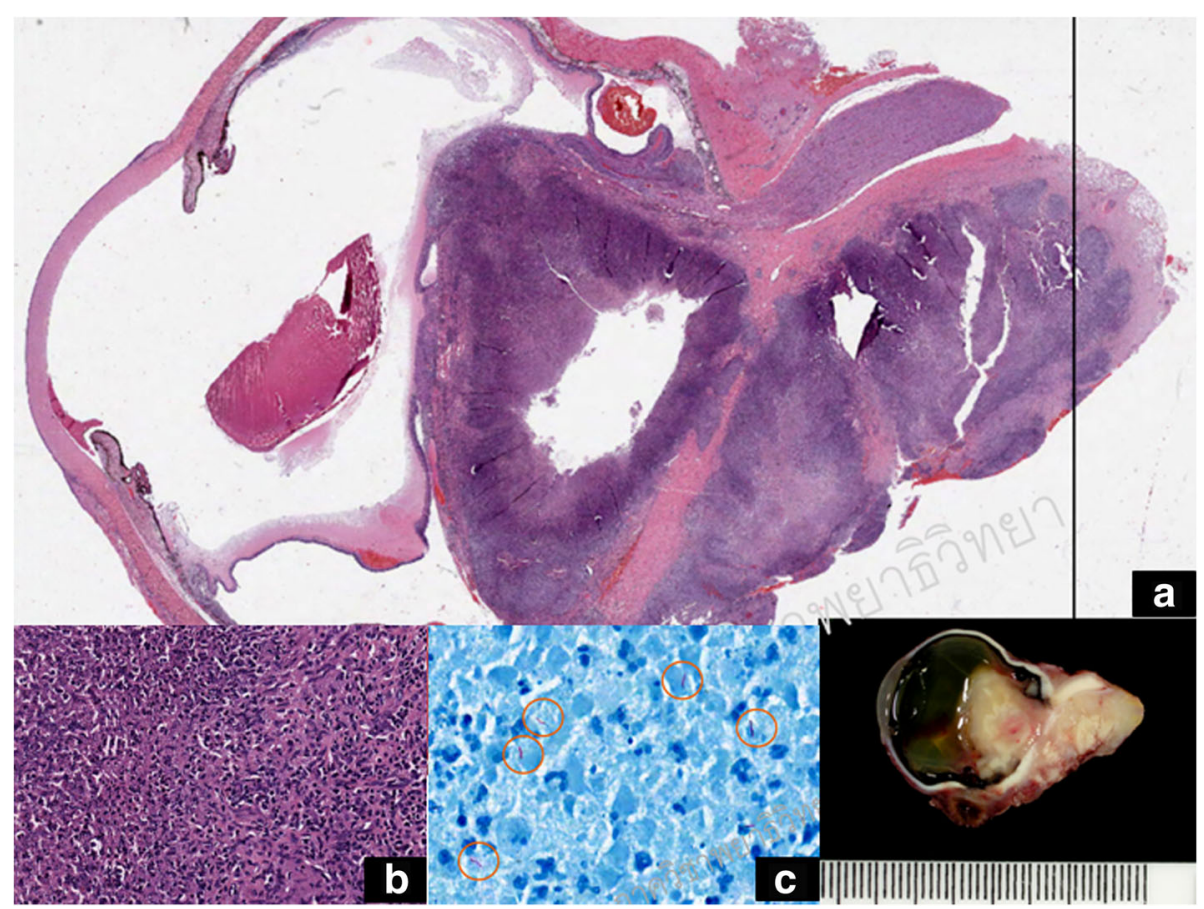

Fig. 2 a A histopathological section of the right eyeball shows an ill-defined, soft, grayish white mass with central necrosis extending from the vitreous cavity into a retrobulbar region, attached to sclera and closed to the optic nerve. $\mathbf{b}$ Microscopic findings revealed granulomatous inflammation with accumulations of predominantly mononuclear cells. c Acid-fast bacilli are shown within orange circles

revealed chronic granulomatous inflammation and caseous necrosis, which was positive for AFB stain and compatible with mycobacterial infection (Fig. 2). Cultured tissue was negative for mycobacterium. Three months later, the result of a conjunctival pus swab culture was positive for drug-susceptible Mtb. The ID specialist recommended continuation of the secondline oral ATT for 18 more months. There was no sign of recurrent tuberculous mycobacterium infection around the exenterated wound at the patient's last visit,

Table 1 Reported cases of Mycobacterium tuberculosis panophthalmitis, orbital abscess, orbital tuberculoma, and orbital apex syndrome with wide varieties in age range, treatment outcomes, and visual prognosis

\begin{tabular}{|c|c|c|c|c|c|c|c|c|c|}
\hline Patient & Age (years) & Sex & Nationality & Health status & Ocular diagnosis & Initial VA & Final VA & Surgical treatment & References \\
\hline 1 & 29 & $\mathrm{~F}$ & Burmese & HIV & $\begin{array}{l}\text { Panophthalmitis with } \\
\text { orbital abscess }\end{array}$ & NPL & - & Subtotal exenteration & Our patient \\
\hline 2 & 73 & M & N/A & Healthy & Panophthalmitis & $6 / 60$ & - & Enucleation & {$[8]$} \\
\hline 3 & 14 & M & N/A & N/A & Panophthalmitis & N/A & N/A & N/A & {$[9]$} \\
\hline 4 & 59 & $\mathrm{~F}$ & Indian & Healthy & Orbital tuberculoma & No visual loss & No visual loss & - & {$[10]$} \\
\hline 5 & 78 & $\mathrm{~F}$ & African & Healthy & Orbital tuberculoma & No visual loss & No visual loss & Anterior orbitotomy & {$[10]$} \\
\hline 6 & 12 & $\mathrm{~F}$ & N/A & N/A & Panophthalmitis & NPL & - & Enucleation & {$[11]$} \\
\hline 7 & 15 & $\mathrm{~F}$ & Indian & N/A & Orbital abscess & $6 / 6$ & $6 / 6$ & Abscess drainage & {$[12]$} \\
\hline 8 & 86 & $\mathrm{~F}$ & Caucasian & N/A & Orbital tuberculoma & Blind & Blind & - & [13] \\
\hline 9 & 6 & M & Indian & N/A & Orbital abscess & $6 / 6$ & $6 / 6$ & FNA & {$[14]$} \\
\hline 10 & 16 & $\mathrm{~F}$ & Afro-Caribbean & N/A & Orbital apex syndrome & $6 / 190$ & PL & - & {$[15]$} \\
\hline 11 & 7 & $\mathrm{~F}$ & N/A & Healthy & Orbital abscess & $6 / 6$ & N/A & Pus evacuation & {$[16]$} \\
\hline 12 & 29 & N/A & N/A & Healthy & Orbital mass & N/A & N/A & - & {$[17]$} \\
\hline 13 & 1 & N/A & Nigerian & N/A & Panophthalmitis & N/A & - & Enucleation & [18] \\
\hline 14 & 27 & M & Indian & HIV & Orbital abscess & NPL & NPL & - & [19] \\
\hline
\end{tabular}

Abbreviations: VA Visual acuity, F Female, M Male, NPL No perception of light, PL Light perception, HIV human immunodeficiency virus infected, FNA Fine-needle aspiration, N/A Not available 
the 6-month follow-up, at Siriraj Hospital. After that visit, she was transferred back to her primary care hospital to complete her course of treatment. Highly active antiretroviral therapy, or HAART, was initiated afterward at the primary care hospital according to the health care policy in Thailand.

\section{Discussion}

Reports of tuberculous panophthalmitis and/or orbital tuberculosis with or without orbital abscess are infrequent. As described in Table 1, many patients have either panophthalmitis or orbital infection, unlike our patient, who had extensive panophthalmitis and orbital abscesses despite 5 months of conventional ATT. Our patient's presentation led to a high suspicion of drugresistant tuberculosis. In a study done in China, researchers reported that the prevalence of any resistance to first-line drugs was $33.2 \%$ and that the prevalence of multidrug-resistant tuberculosis (MDR-TB) was 5.7\% [3]. Wilson and Tsukayama reported risk factors for the development and perpetuation of drug-resistant Mtb, as shown in Table 2 [4]. Our patient had drugsusceptible Mtb; thus, drug resistance was not responsible for the poor treatment outcome in her case. Poor ATT compliance was likely the main causative factor. Directly observed treatment, short course (DOTS), especially with an individualized treatment regimen, is considered a successful method to improve cure rate and treatment completion in many centers [5]; moreover, DOTS can also prevent the development of drug resistance [6]. Statistical analysis to evaluate associated risk factors for developing tuberculous mycobacterial orbital abscess and/or panophthalmitis and visual prognosis has not been achievable, owing to the small number of cases in this patient population. For patients with HIV infection, debate continues regarding

Table 2 Risk factors for the development of drug resistance to Mycobacterium tuberculosis as reported by Wilson and Tsukayama [4]

Previous treatment for Mtb

Prolonged hospitalization (in Mtb-endemic regions)

human immunodeficiency virus coinfection

Inappropriate prescribing of combination ATT (incorrect drug selection, dosing, and improper dispensing)

Lack of directly observed therapy use during therapy and subsequent patient noncompliance with prescribed therapy

Lack of sustainable drug availability to patients (for example, second-line drugs for drug-resistant tuberculosis) through an inadequate pharmaceutical supply chain or failure to provide free treatment

Overuse of fluoroquinolones in other non-Mtb respiratory infection syndromes that propagates fluoroquinolone-resistant Mtb

Delays in diagnosing drug-resistant Mtb

Abbreviations: Mtb Mycobacterium tuberculosis, ATT Antituberculous therapy the ideal timing of HAART initiation and concomitant administration with ATT. The advantages of early HAART administration include higher cure rates, reduced risk of relapse, reduced risk of other HIVassociated opportunistic infections, and lower mortality rates. On the other hand, the disadvantages of early HAART initiation include potential drug interaction with rifampicin, thus limiting coadministration of selected protease inhibitors. This could result in cumulative toxicity, therapeutic failure, and risk of immune reconstitution inflammatory syndrome $[2,7]$. Our patient had not received HAART until almost 1 year after she was first diagnosed with ЕРTB infection.

\section{Conclusions}

Close and careful observation of patients undergoing ATT is absolutely essential. Poor compliance is one of the main risk factors for developing MDR-TB. Even in cases where there is no drug resistance, poor compliance can alter treatment outcome and prognosis, as evidenced by the course and outcome of our patient.

\section{Acknowledgements \\ The authors acknowledge Wilawan Sanpan for preparation of the manuscript for submission. \\ Funding \\ This is an unfunded report. \\ Availability of data and materials \\ Please contact the corresponding author for data requests. \\ Authors' contributions \\ All authors made substantial contributions to the case report's conception and design, acquisition of data, and analysis and interpretation of data. PC and SB were involved in drafting the manuscript and revising it critically for important intellectual content. PC gave final approval of the version to be published. All authors read and approved the final manuscript.}

\section{Competing interests}

The authors declare that they have no competing interests.

\section{Consent for publication}

Written informed consent was obtained from the patient for publication of this case report and any accompanying images. A copy of the written consent is available for review by the Editor-in-Chief of this journal.

Ethics approval and consent to participate

The need for ethics approval was waived by the Siriraj Institutional Review Board, Faculty of Medicine, Mahidol University.

\section{Publisher's Note}

Springer Nature remains neutral with regard to jurisdictional claims in published maps and institutional affiliations.

\section{Author details}

${ }^{1}$ Department of Ophthalmology, Faculty of Medicine, Siriraj Hospital, Mahidol University, 2 Wanglang Road, Bangkoknoi, Bangkok 10700, Thailand. 2Department of Pathology, Faculty of Medicine, Siriraj Hospital, Mahidol University, Bangkok, Thailand. ${ }^{3}$ Department of Ophthalmology, Faculty of Medicine, Thammasat Hospital, Thammasat University, Bangkok, Thailand. 
Received: 14 December 2016 Accepted: 31 May 2017

\section{Published online: 04 July 2017}

\section{References}

1. Gupta A, Sharma A, Bansal R, Sharma K. Classification of intraocular tuberculosis. Ocul Immunol Inflamm. 2015;23(1):7-13.

2. Montales MT, Chaudhury A, Beebe A, Patil S, Patil N. HIV-associated TB syndemic: a growing clinical challenge worldwide. Front Public Health. 2015;3:281.

3. Wang SF, Zhou Y, Pang Y, Zheng HW, Zhao YL. Prevalence and risk factors of primary drug-resistant tuberculosis in China. Biomed Environ Sci. 2016;29(2):91-8.

4. Wilson JW, Tsukayama DT. Extensively drug-resistant tuberculosis: principles of resistance, diagnosis, and management. Mayo Clin Proc. 2016;91(4):482-95.

5. Kibret KT, Moges Y, Memiah P, Biadgilign S. Treatment outcomes for multidrug-resistant tuberculosis under DOTS-Plus: a systematic review and meta-analysis of published studies. Infect Dis Poverty. 2017;6(1):7.

6. McLaren ZM, Milliken AA, Meyer AJ, Sharp AR. Does directly observed therapy improve tuberculosis treatment? More evidence is needed to guide tuberculosis policy. BMC Infect Dis. 2016;16(1):537.

7. Havlir DV, Currier JS. CROI 2015: complications of HIV infection and antiretroviral therapy. Top Antivir Med. 2015;23(1):56-65.

8. Arrell RW. Acute tuberculous panophthalmitis. Arch Ophthalmol. 1967;78(1):51-4.

9. Wadhwani M, Sethi S, Beri S, Jain R, Dsouza P, Nangia A, et al. An unusual case of metastatic tubercular panophthalmitis in a 14-year-old boy. J Pediatr Ophthalmol Strabismus. 2011;48(5):318-9.

10. Salam T, Uddin JM, Collin JR, Verity DH, Beaconsfield M, Rose GE. Periocular tuberculous disease: experience from a UK eye hospital. Br J Ophthalmol. 2015;99(5):582-5.

11. Chawla R, Garg S, Venkatesh P, Kashyap S, Tewari HK. Case report of tuberculous panophthalmitis. Med Sci Monit. 2004;10(10):CS57-9.

12. Babu K, Mukhopadhyay M, Bhat SS, Chinmayee J. Orbital and adnexal tuberculosis: a case series from a South Indian population. J Ophthalmic Inflamm Infect. 2014;4:12.

13. Benech N, Jouanneau E, Chidiac C, Ferry T. Orbital granuloma: a rare manifestation of extrapulmonary tuberculosis. BMJ Case Rep. 2016;2016. doi: 10.1136/bcr-2016-214542.

14. Sharma K, Kanaujia V, Jain A, Bains S, Suman S. Tuberculous orbital abscess associated with thyroid tuberculosis. J Ophthalmic Vis Res. 2011;6(3):204-7.

15. Tanawade RG, Thampy RS, Wilson S, Lloyd IC, Ashworth J. Tuberculous orbital apex syndrome with severe irreversible visual loss. Orbit. 2015;34(3):172-4.

16. Aggarwal D, Suri A, Mahapatra AK. Orbital tuberculosis with abscess. J Neuroophthalmol. 2002;22(3):208-10.

17. de de Carvalho Santana R, Louzada Jr P, Bollela VR, Cruz AA, Fonseca BA Orbital tuberculosis presenting as proptosis and fever: the risk of empiric corticosteroids. Int Ophthalmol. 2014;34(1):133-6.

18. McMoli TE, Mordi VP, Grange A, Abiose A. Tuberculous panophthalmitis. J Pediatr Ophthalmol Strabismus. 1978;15(6):383-5.

19. Banait S, Jain J, Parihar PH, Karwassara V. Orbital tuberculosis manifesting as proptosis in an immunocompromised host. Indian J Sex Transm Dis. 2012;33(2):128-30

\section{Submit your next manuscript to BioMed Central and we will help you at every step:}

- We accept pre-submission inquiries

- Our selector tool helps you to find the most relevant journal

- We provide round the clock customer support

- Convenient online submission

- Thorough peer review

- Inclusion in PubMed and all major indexing services

- Maximum visibility for your research

Submit your manuscript at www.biomedcentral.com/submit 